\title{
Análisis de sincronización de semáforos utilizando el programa Synchro
}

\author{
Synchronization analysis of traffic lights using the software Synchro
}

\section{Dra. María Liliana Alba Menéndez.}

Instituto Superior Politécnico de Tecnologías y Ciencias, ISPTEC, Luanda, Angola

mlalbam@gmail.com
Dra. Oisy Hernández Menéndez.

Universidad Federal del Oeste de la Bahía, Bahía, Brasil

oisy.menendez@ufob.edu.br

Fecha de recepción: 02 de marzo de 2020 / Fecha de aprobación: 03 de julio de 2020

\section{RESUMEN}

La congestión del tránsito es un problema que se manifiesta a nivel internacional y del cual Cuba no está exenta, a pesar de que los niveles existentes no llegan a los presentados por los países con alto nivel de motorización. Las intersecciones semaforizadas de las arterias urbanas de La Habana son las más congestionadas, donde se reflejan impactos negativos como accidentes de tránsito, demoras, colas y emisión de contaminantes a la atmósfera. Una de las medidas para mitigar la congestión es coordinar las cadenas semafóricas, lo que clasifica como una importante medida de bajo costo. En esta investigación se aborda el tema de la evaluación de la eficiencia de cadenas sincronizadas utilizando parámetros generales como tiempo de ciclo, intervalos de verde, desfasaje y ancho de banda, teniendo en cuenta la minimización de las demoras de los usuarios, utilizando el software Synchro, y se exponen los resultados obtenidos para un caso de estudio.

Palabras clave: intersecciones, sincronización, demoras, nivel de servicio.

\section{ABSTRACT}

Traffic congestion is a problem that occurs worldwide, and Cuba is not exempt, even when the existing levels do not reach those reported by countries with high level of motorization. Signalized intersections in urban arteries of Havana are the most congested, which reflects negative impacts such as traffic accidents, delays, queue, and emission of pollutants into the atmosphere. A possible low-cost solution to decrease congestion is to coordinate the group of traffic signals. Therefore, this research aims to assess the efficiency of synchronized channels using parameters as cycle time, green intervals, and bandwidth for a case study using Synchro software. This research does not consider the minimization of delays of the users. The results support the feasibility of using these parameters to evaluate and select the design holding the most effective synchronization.

Keywords: intersections, coordination, delays, level of service. 


\section{INTRODUCCIÓN}

Actualmente, los mayores problemas de congestión en las redes viales urbanas se producen en las intersecciones semaforizadas, debido fundamentalmente al aumento vertiginoso del parque vehicular, sobre todo en el último siglo (Pirez, 2000; CNIT, 2010; Jiménez García, Medina Flores y Figueroa Fernández, 2015), lo que hizo necesario desarrollar modelos matemáticos capaces de generar soluciones viales adecuadas a las características del flujo vehicular (Osorio et al., 2015; Nyame-Baafi, Adams y Osei, 2018).

Con el aumento exponencial del volumen de tráfico se incrementa el número de intersecciones reguladas por semáforos, lo que hace necesaria una sincronización, tanto más precisa cuanto más cercana a la saturación se encuentre la arteria puesto que, en estos casos, pequeños errores de cálculo conducirían al bloqueo de la misma con el consiguiente aumento desmesurado de los tiempos de recorrido (Díaz et al., 2002).

La sincronización de semáforos mejora potencialmente las operaciones de tráfico reduciendo significativamente las demoras (ATAC, 2003; Trafficware, 2017), produciendo ahorros de tiempo, combustible y contaminación (Koonce et al., 2008; Molina-Navarro, Zamora-Castro, Remess-Pérez y Lagunes-Lagunes 2016). En los últimos 30 años se ha producido un extraordinario desarrollo tecnológico que, mediante la incorporación de la computación y la electrónica en el manejo de complejas situaciones de tránsito, ha permitido el control de amplias redes, con sistemas centralizados y flexibles en relación con la demanda. Los programas como VISSIM, SIDRA, Synchro, AIMSUM, Simtraffic, CORSIM y TRANSYT han cambiado completamente la forma de enfrentar el problema, ya que proveen capacidades para avanzar en la optimización de los sistemas de tránsito (Gettman y Head, 2003; Mahmud, Ferreira, Hoque y Tavassoli, 2019).

La simulación del tránsito utilizando programas de modelación permite la evaluación del tráfico real y la evaluación de los parámetros de eficiencia de las intersecciones semaforizadas y no semaforizadas, para proponer estrategias que posibiliten la optimización del control del tránsito en las intersecciones (Araujo, Jacondino y Cybis, 2004; Tianzi, Shaochen y Hongxu, 2013; de Araújo y de Castro Neto, 2018; Mahmud et al., 2019). El programa TRANSYT, ha sido ampliamente probado en muchos países, y ha llegado a constituir prácticamente un estándar internacional para la sincronización de redes (Shaaban y Radwan, 2005; Traffic Engineering Division, 2005).
Otros programas que se muestran factibles para coordinar semáforos de tiempo fijo son Promodel (Espinoza Mondragón, Jiménez García, Medina Flores y Yáñez Contreras, 2015; Jiménez García et al., 2015), CORSIM, PASSER (Benekohal, Elzohairy y Saak, 2001) y el Synchro (ATAC, 2003; Park y Chen, 2010; Trafficware, 2017).

A pesar de los esfuerzos realizados en los últimos años para la mejora de la circulación, la vialidad urbana cubana presenta todavía deficiencias que se hace imprescindible corregir; se debe mejorar el diseño de las intersecciones, corregir el ciclo de los semáforos, coordinar intersecciones semafóricas en arterias principales, entre otros (CNIT, 2010).

Para enfrentar el reto anterior, las autoridades están tomando importantes medidas tales como la señalización horizontal y vertical de las vías principales, la sustitución de los controles semafóricos por equipos modernos -que en muchos casos cuentan con contadores regresivos que indican a los conductores el tiempo que resta para el cambio de luz, disminuyendo el tiempo perdido y el estrés del conductor y, por lo tanto, aumentando la seguridad vial-, adecuando las temporizaciones a los volúmenes de demanda y sincronizando el movimiento en las principales arterias, siendo esta última acción una de las soluciones viales de más bajo costo que se implementa (Gil, 2012).

Para el diseño del sincronismo en Cuba se utilizan las técnicas tradicionales de cálculo manual basado en criterios puramente geométricos, obteniendo parámetros como tiempo de ciclo, intervalos de verde, desfasaje y ancho de banda que se suele conocer como "onda verde" (Cardoso Espinosa y Moreno, 2012). En algunos casos, se emplea el software Trafficware (Synchro Studio versión 10.1 con revisión 1 (10.1.1.1) de Trafficware LLC (2017)), el cual permite calcular indicadores de eficiencia por el método del Transportation Research Board (2010) y Webster (1958) y, además, evalúa los beneficios obtenidos por disminución de demora, gasto de combustible y emisión de contaminantes. Es muy factible para realizar estudios antes-después mediante simulación sin intervenir en el terreno, ahorrando recursos y evaluando variantes para implementar la más efectiva (Shaaban y Radwan, 2005; Hajbabaie, Medina y Bnekohal, 2011; Trafficware, 2017).

Por otro lado, el Centro Nacional de Ingeniería de Tránsito de Cuba y el Complejo de Investigaciones Tecnológicas Integradas, en conjunto con el Departamento de Automática del ISPJAE, desarrollaron un sistema inteligente de transporte para el control del flujo vehicular y el diseño total de controladores semafóricos. El software muestra como salida 
una simulación del sincronismo mediante una gráfica $y$, además, genera un reporte que informa datos generales de las intersecciones y su plan de tiempo respectivo (Cardoso Espinosa y Moreno, 2012).

En La Habana, capital de Cuba, se concentran los mayores volúmenes de tránsito en las principales arterias, que son: Avenida de Rancho Boyeros, 5ta Avenida, Paseo, Avenida 23, Avenida 26, Salvador Allende y Reina (Alba, 2008). La 5 ta Avenida, compuesta por 7 intersecciones semaforizadas tiene problemas de congestión, altos volúmenes de demanda que provocan largas colas y altas demoras, motivo por el cual se realizó una evaluación de las diferentes variantes de sincronización, como cadenas aisladas o en red, con el objetivo de mejorar las condiciones de circulación de la arteria, buscando aumentar la velocidad, mejorar los niveles de servicio de la arteria y sus intersecciones, para disminuir las demoras y colas actuales.

\section{MÉTODOS EMPLEADOS EN EL ESTUDIO}

Cuando las intersecciones semaforizadas de una arteria se encuentran cercanas y los volúmenes de tránsito son altos, los sistemas de semáforos coordinados son preferibles al trabajo como intersecciones aisladas. La sincronización consiste en programar el encendido de las luces verdes de los semáforos de tal forma que los vehículos puedan atravesar una vía, de extremo a extremo, a una velocidad constante y sin detenerse, procurando que las interrupciones generadas por la luz roja sean mínimas. Es una de las formas más eficientes de reducir demoras, consumo de combustible, contaminación y accidentes (Bezerra, 2007; Koonce et al., 2008).

Los parámetros más importantes que deben considerarse para la sincronización de un sistema semafórico son: el ciclo, el reparto o distribución de tiempos en verde de cada fase, y el desfase, siendo este último parámetro el período de tiempo que transcurre entre el comienzo de una fase específica en un semáforo y su comienzo en la intersección siguiente (Díaz y Díaz, 2002; Bezerra, 2007; Koonce et al., 2008). El Manual de dispositivos de control del tránsito (Koonce et al., 2008) y las regulaciones cubanas recomiendan que las intersecciones semaforizadas cercanas entre sí a 800 metros o menos deben ser sincronizadas con un ciclo común (CNIT, 2008).

Para diseñar la sincronización de intersecciones semaforizadas existen varios métodos, entre los que destaca el conocido como geométrico, ya que maximiza el ancho de banda verde utilizando un diagrama espacio-tiempo al fijar una velocidad de circulación y un tiempo de ciclo (Cal, Mayor y Cárdenas, 2010; Koonce et al., 2008). Se utiliza para coordinaciones con índice de saturación bajo (relación volumen/capacidad) y el usuario aprecia directamente las ventajas de la sincronización.

El otro método se basa en la optimización de las variables de tráfico, en específico en las demoras. Por su complejidad se utilizan herramientas computacionales especializadas (softwares), que cuentan con complejos algoritmos matemáticos muy utilizados en cadenas semaforizadas próximas a la saturación, para obtener la máxima capacidad posible.

Este último será el método por utilizar en la investigación, auxiliándose del software Synchro, un programa ampliamente utilizado por organismos e instituciones de tráfico debido a las grandes ventajas que ofrece, entre ellas, la simulación de las coordinaciones de tráfico existente en una red vial, contando con una variedad de parámetros de eficiencia ligados a un reporte gráfico que permite valorar de manera directa qué tan aproximados son los resultados de campo. Evalúa y optimiza planes de sistemas sincronizados basados en los volúmenes de tránsito y las condiciones geométricas existentes y sus modelos pueden ser utilizados para predecir el comportamiento esperado del sistema (ATAC, 2003; Park y Chen, 2010; Trafficware, 2017).

Con la utilización del Synchro se evalúan los siguientes parámetros de eficiencia que caracterizan a las cadenas sincronizadas, para definir la variante que mayores beneficios proporciona: factor de coordinabilidad (FC), calidad de la progresión, maximización del ancho de banda, vehículos en la zona de dilema, demoras, nivel de servicio, relación volumen/ capacidad (v/c), paradas totales y paradas por vehículos.

\section{CASO DE ESTUDIO}

\subsection{Caracterización de la arteria 5ta Avenida en La Habana, Cuba}

La 5ta Avenida constituye una arteria principal de La Habana, ubicada en el litoral noroeste que, por su origen y destino, forma parte de uno de los ejes viales con función de movilidad más importantes al enlazar de forma directa las zonas este y oeste (Alba, 2008). Con una longitud de cinco kilómetros, presenta siete intersecciones controladas por semáforos, con altos volúmenes de demanda y tráfico pendular que, en la hora pico de la mañana, se mueve en dirección hacia el centro de la ciudad. En la Figura 1 se muestra su trazado y las intersecciones semaforizadas principales que la conforman. 
Se realizaron estudios de volúmenes vehiculares en las intersecciones semaforizadas y sus incorporaciones desde las vías transversales, de las características geométricas y del diseño de fases y reglaje existente en cada semáforo.

Partiendo de los resultados, la hora pico se ubicó en el intervalo de 7:30 a 8:30 am. En el Cuadro 1 se muestran las características de circulación de las intersecciones que componen la arteria, específicamente la pendiente (Pend.), los volúmenes por movimiento (veh/h), factor hora pico (FHP) y porcentaje de vehículos pesados (\% VP).

El plan de fases existente en toda la arteria se presenta en el Cuadro 2. El ciclo natural de la arteria es de 100 segundos.

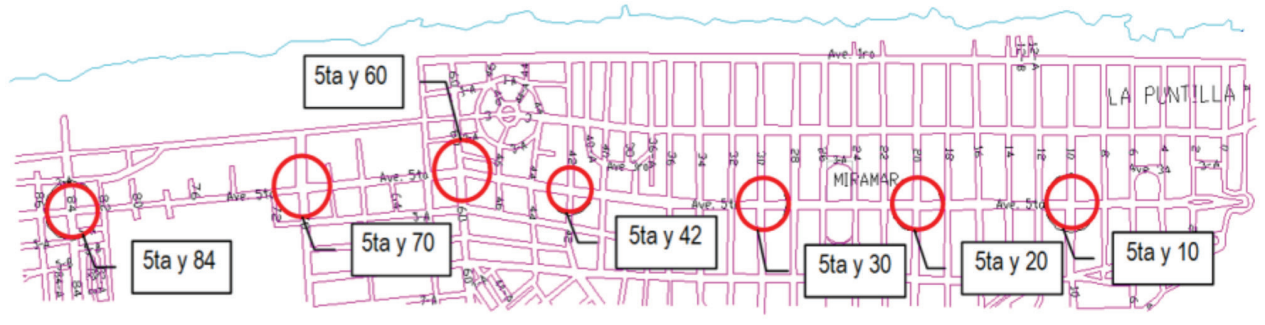

Figura 1. Trazado de 5ta Avenida y sus intersecciones principales

Cuadro 1. Resumen de las características de circulación de 5ta Avenida

\begin{tabular}{|c|c|c|c|c|c|c|c|c|}
\hline \multirow{2}{*}{ Intersecciones } & \multirow{2}{*}{ Acceso } & \multirow{2}{*}{ Pend. (\%) } & \multicolumn{4}{|c|}{ Volúmenes en la hora pico (veh/h) } & \multirow{2}{*}{ FHP } & \multirow{2}{*}{$\%$ VP } \\
\hline & & & lzq. & Recto & Der. & Total & & \\
\hline \multirow{4}{*}{ 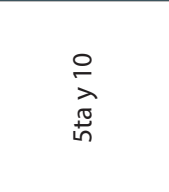 } & Desde este & 1 & & 964 & 33 & \multirow{4}{*}{3171} & 0,99 & 0 \\
\hline & Desde oeste & 1 & & 1080 & 108 & & 0,98 & 0 \\
\hline & Desde norte & 1 & 110 & 346 & 20 & & 0,93 & 9 \\
\hline & Desde sur & -1 & 157 & 298 & 55 & & 0,93 & 4 \\
\hline \multirow{4}{*}{ 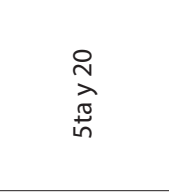 } & Desde este & 1 & & 838 & 71 & \multirow{4}{*}{2556} & 0,94 & 0 \\
\hline & Desde oeste & 1 & & 1080 & 56 & & 0,91 & 0 \\
\hline & Desde norte & 1 & 72 & 82 & 7 & & 0,95 & 6 \\
\hline & Desde sur & -2 & 72 & 249 & 29 & & 0,93 & 3 \\
\hline \multirow{4}{*}{$\underset{\substack{n \\
\stackrel{D}{D}}}{i n}$} & Desde este & 1 & & 1016 & 21 & \multirow{4}{*}{2650} & 0,98 & 0 \\
\hline & Desde oeste & 1 & & 901 & 60 & & 0,94 & 0 \\
\hline & Desde norte & 1 & 63 & 125 & 24 & & 0,89 & 8 \\
\hline & Desde sur & -2 & 106 & 244 & 90 & & 0,90 & 4 \\
\hline \multirow{4}{*}{$\begin{array}{l}\mathcal{F} \\
\underset{\sigma}{\sim} \\
\stackrel{ే}{n}\end{array}$} & Desde este & 1 & & 970 & 20 & \multirow{4}{*}{2648} & 0,98 & 0 \\
\hline & Desde oeste & 1 & & 1096 & 92 & & 0,97 & 0 \\
\hline & Desde norte & 1 & 24 & 129 & 7 & & 0,95 & 3 \\
\hline & Desde sur & -2 & 178 & 110 & 22 & & 0,98 & 1 \\
\hline \multirow{4}{*}{ 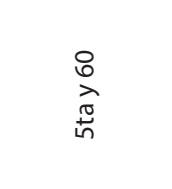 } & Desde este & 1 & & 1050 & 28 & \multirow{4}{*}{2709} & 0,93 & 0 \\
\hline & Desde oeste & 1 & & 1102 & 35 & & 0,94 & 0 \\
\hline & Desde norte & 1 & 42 & 118 & 19 & & 0,99 & 9 \\
\hline & Desde sur & -4 & 90 & 192 & 33 & & 0,97 & 6 \\
\hline \multirow{4}{*}{ 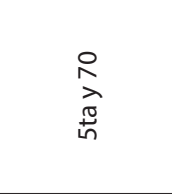 } & Desde este & -1 & & 1062 & 255 & \multirow{4}{*}{3168} & 0,92 & 0 \\
\hline & Desde oeste & -1 & & 1120 & 97 & & 0,97 & 0 \\
\hline & Desde norte & -1 & 156 & 218 & 16 & & 0,89 & 12 \\
\hline & Desde sur & -5 & 156 & 59 & 29 & & 0,93 & 8 \\
\hline \multirow{4}{*}{$\underset{\stackrel{D}{D}}{\stackrel{D}{D}}$} & Desde este & 2 & & 767 & 66 & \multirow{4}{*}{3109} & 0,90 & 0 \\
\hline & Desde oeste & -4 & & 1191 & 208 & & 0,94 & 0 \\
\hline & Desde norte & -1 & 46 & 160 & 12 & & 0,95 & 5 \\
\hline & Desde sur & -3 & 323 & 276 & 60 & & 0,97 & 3 \\
\hline
\end{tabular}


Cuadro 2. Plan de fases en la arteria

\begin{tabular}{|c|c|c|}
\hline Fase & Descripción & Movimientos \\
\hline 1 & 5ta Avenida hacia el este y hacia el oeste & Recto y derecha \\
\hline 2 & Calle transversal (hacia el norte) & Izquierda, recto y derecha \\
\hline 3 & Calle transversal (hacia el sur) & Izquierda, recto y derecha \\
\hline
\end{tabular}

\subsection{Evaluación de las condiciones de circulación antes de la sincronización}

La utilización del Synchro permite obtener las principales medidas de efectividad para las intersecciones semaforizadas y las arterias. Las medidas de efectividad de demora, nivel de servicio (NS) y relación volumen/capacidad (v/c) obtenidas para las intersecciones que componen la 5ta Avenida se muestran en los Cuadros 3 y 4 . Los resultados confirman los problemas de congestión y demoras detectados durante los estudios realizados in situ.

Cuadro 3. Parámetros de eficiencia para las intersecciones semaforizadas de 5 ta Avenida antes del sincronismo

\begin{tabular}{|c|c|c|c|c|c|c|}
\hline \multirow{2}{*}{$\begin{array}{c}\text { Acceso } \\
\text { Intersecciones }\end{array}$} & \multicolumn{3}{|c|}{$\begin{array}{l}\text { 5ta Avenida } \\
\text { descle este }\end{array}$} & \multicolumn{3}{|c|}{$\begin{array}{l}\text { 5ta Avenida } \\
\text { desde oeste }\end{array}$} \\
\hline & Demora (s/veh) & NS & $\mathrm{v} / \mathrm{c}$ & Demora (s/veh) & NS & $\mathrm{v} / \mathrm{c}$ \\
\hline 5 ta y 10 & 20,10 & $\mathrm{C}$ & 0,68 & 25,70 & C & 0,86 \\
\hline 5 ta y 20 & 11,30 & B & 0,82 & 40,00 & $\mathrm{D}$ & 0,87 \\
\hline 5 ta y 30 & 6,60 & $A$ & 0,79 & 18,40 & B & 0,85 \\
\hline 5 ta y 42 & 22,80 & $\mathrm{C}$ & 0,74 & 25,10 & C & 0,80 \\
\hline 5 ta y 60 & 31,10 & $\mathrm{C}$ & 0,79 & 24,70 & C & 0,92 \\
\hline 5 ta y 70 & 17,40 & $\mathrm{~B}$ & 0,87 & 27,50 & C & 0,84 \\
\hline 5 ta y 84 & 31,50 & C & 0,85 & 95,40 & $\mathrm{~F}$ & 1,14 \\
\hline
\end{tabular}

Cuadro 4. Indicadores de eficiencia y nivel de servicio arterial en 5ta Avenida antes de coordinar

\begin{tabular}{|c|c|c|c|c|c|}
\hline Sentido & $\begin{array}{l}\text { Tiempo de } \\
\text { marcha (s) }\end{array}$ & $\begin{array}{l}\text { Demora } \\
\text { (s/veh) }\end{array}$ & Tiempo de recorrido (s) & $\begin{array}{c}\text { Velocidad } \\
\text { arterial (km/h) }\end{array}$ & NS \\
\hline Desde este & 210,8 & 140,8 & 351,6 & 39,2 & $\mathrm{D}$ \\
\hline Desde oeste & 206,4 & 256,8 & 463,2 & 29,2 & $\mathrm{E}$ \\
\hline
\end{tabular}


Se evidencia que los niveles de servicio por 5 ta Avenida son aparentemente buenos, lo que está condicionado por el alto valor de la relación tiempo de verde/tiempo total de ciclo, permitiendo que la mayoría de los vehículos que arriban se despejen en cada ciclo. Esto ocasiona un nivel de servicio aceptable en las vías transversales ya que no alcanzan, en ningún caso, el nivel C. Se evaluó además la calidad de la progresión, siendo de baja calidad para la mayoría de las intersecciones, lo que incide en el aumento de las demoras y las colas.

\section{PRESENTACIÓN Y ANÁLISIS DE LOS RESULTADOS}

Para evaluar la conveniencia de coordinar la cadena se utilizó el factor de coordinabilidad, que es una medida de la factibilidad de coordinar las intersecciones en una arteria. Este factor arroja una puntuación entre de 0 y 100, mientras mayor sea su valor mayor será la garantía de mejora en la circulación mediante esta acción. Los factores de coordinabilidad en cada arco de la arteria 5 ta Avenida son mostrados en el Cuadro 5. Es posible observar valores elevados de este factor, lo que permite reafirmar que es muy conveniente coordinar estas intersecciones.
Cuadro 5. Factor de coordinabilidad en 5ta avenida

\begin{tabular}{|c|c|}
\hline Arco & Factor de coordinabilidad \\
\hline De 10 a 20 & 85 \\
\hline De 20 a 30 & 88 \\
\hline De 30 a 42 & 80 \\
\hline De 42 a 60 & 100 \\
\hline De 60 a 70 & 86 \\
\hline De 70 a 84 & 72 \\
\hline
\end{tabular}

\subsection{Variantes para realizar la sincronización de la arteria}

Se analizaron las siguientes variantes de sincronización: para velocidades de 80 y $70 \mathrm{~km} / \mathrm{h}$ con todos los movimientos existentes permitidos y ciclo natural de 100 segundos (variantes 1 y 2). Para las mismas velocidades y movimientos con ciclo optimizado de 94 segundos (variantes 3 y 4). Los parámetros de eficiencia arterial para cada variante se muestran en el Cuadro 6.

Cuadro 6. Indicadores de eficiencia y nivel de servicio arterial para cada variante

\begin{tabular}{|c|c|c|c|c|c|c|c|}
\hline Variante & $\begin{array}{l}\text { Sentido de } \\
\text { circulación }\end{array}$ & $\begin{array}{l}\text { Velocidad de sincronismo } \\
\qquad(\mathrm{km} / \mathrm{h})\end{array}$ & Ciclo (s) & $\begin{array}{l}\text { Demora } \\
\text { (s/veh) }\end{array}$ & $\begin{array}{l}\text { Velocidad } \\
\text { media marcha } \\
(\mathrm{km} / \mathrm{h})\end{array}$ & NS & Ancho de banda (s) \\
\hline \multirow{2}{*}{1} & $\begin{array}{l}\text { Desde } \\
\text { este }\end{array}$ & \multirow{2}{*}{80} & \multirow{2}{*}{100} & 72,90 & 44,80 & $\mathrm{~B}$ & 26 \\
\hline & $\begin{array}{l}\text { Desde } \\
\text { oeste }\end{array}$ & & & 78,00 & 45,50 & $\mathrm{~B}$ & 26 \\
\hline \multirow{2}{*}{2} & $\begin{array}{l}\text { Desde } \\
\text { este }\end{array}$ & \multirow{2}{*}{70} & \multirow{2}{*}{100} & 108,30 & 40,50 & $C$ & 15 \\
\hline & $\begin{array}{l}\text { Desde } \\
\text { oeste }\end{array}$ & & & 99,60 & 41,80 & $C$ & 17 \\
\hline \multirow{2}{*}{3} & $\begin{array}{l}\text { Desde } \\
\text { este }\end{array}$ & \multirow{2}{*}{80} & \multirow{2}{*}{94} & 89,1 & 43,1 & $C$ & 13 \\
\hline & $\begin{array}{l}\text { Desde } \\
\text { oeste }\end{array}$ & & & 85,1 & 43,8 & $C$ & 14 \\
\hline \multirow{2}{*}{4} & $\begin{array}{c}\text { Desde } \\
\text { este }\end{array}$ & \multirow{2}{*}{70} & \multirow{2}{*}{94} & 96,00 & 42,10 & $C$ & 18 \\
\hline & $\begin{array}{l}\text { Desde } \\
\text { oeste }\end{array}$ & & & 106,70 & 40,90 & $C$ & 16 \\
\hline
\end{tabular}


Del cuadro anterior se puede concluir que la variante de solución para lograr la mejor circulación de la arteria 5ta Avenida es la variante de sincronización 1 , porque presenta el mejor nivel de servicio, las menores demoras, la mayor velocidad arterial y los mayores anchos de bandas para cada sentido.

$\mathrm{Si}$ comparamos los resultados obtenidos en la variante seleccionada con los del Cuadro 4, se puede observar que los indicadores de eficiencia mejoran considerablemente con el sincronismo, en especial las demoras (en el sentido desde el este se disminuyen aproximadamente el 50\% de las demoras que existen actualmente, y en el sentido desde el oeste se disminuye hasta un 70\%). El nivel de servicio arterial se mejora de $\mathrm{D}$ o E para B en ambos sentidos de circulación. Por otro lado, la velocidad arterial también aumentó en 5,6 km/h en el sentido desde el este y $16,3 \mathrm{~km} / \mathrm{h}$ en el sentido desde el oeste.

El diagrama de bandas que proporciona el software para la variante seleccionada se muestra en la Figura 2.

\subsection{Calidad de la progresión}

La calidad de la progresión caracteriza el movimiento de los pelotones de vehículos desde una intersección semaforizada a la próxima, lo que afecta en la práctica la apreciación por el usuario de la calidad de la operación. La calidad de la progresión se evalúa a través del parámetro relación de columna (Rc), como una función del porcentaje de vehículos que no se detienen en la intersección, empleando la Ecuación 1.

$$
R c=P V V\left(\frac{c}{g}\right)
$$

donde

Rc = Relación de columna .

PVV = Porcentaje de vehículos que pasan con luz verde sin detenerse (\%).

$\mathrm{C}=$ Tiempo total de ciclo (s).

$g$ = Tiempo de verde efectivo por la vía sincronizada (s).

A partir de los cálculos de Rc se evaluó la calidad de la progresión para cada intersección con la cadena sincronizada (Transportation Research Board, 2010) que se muestra en el Cuadro 7.

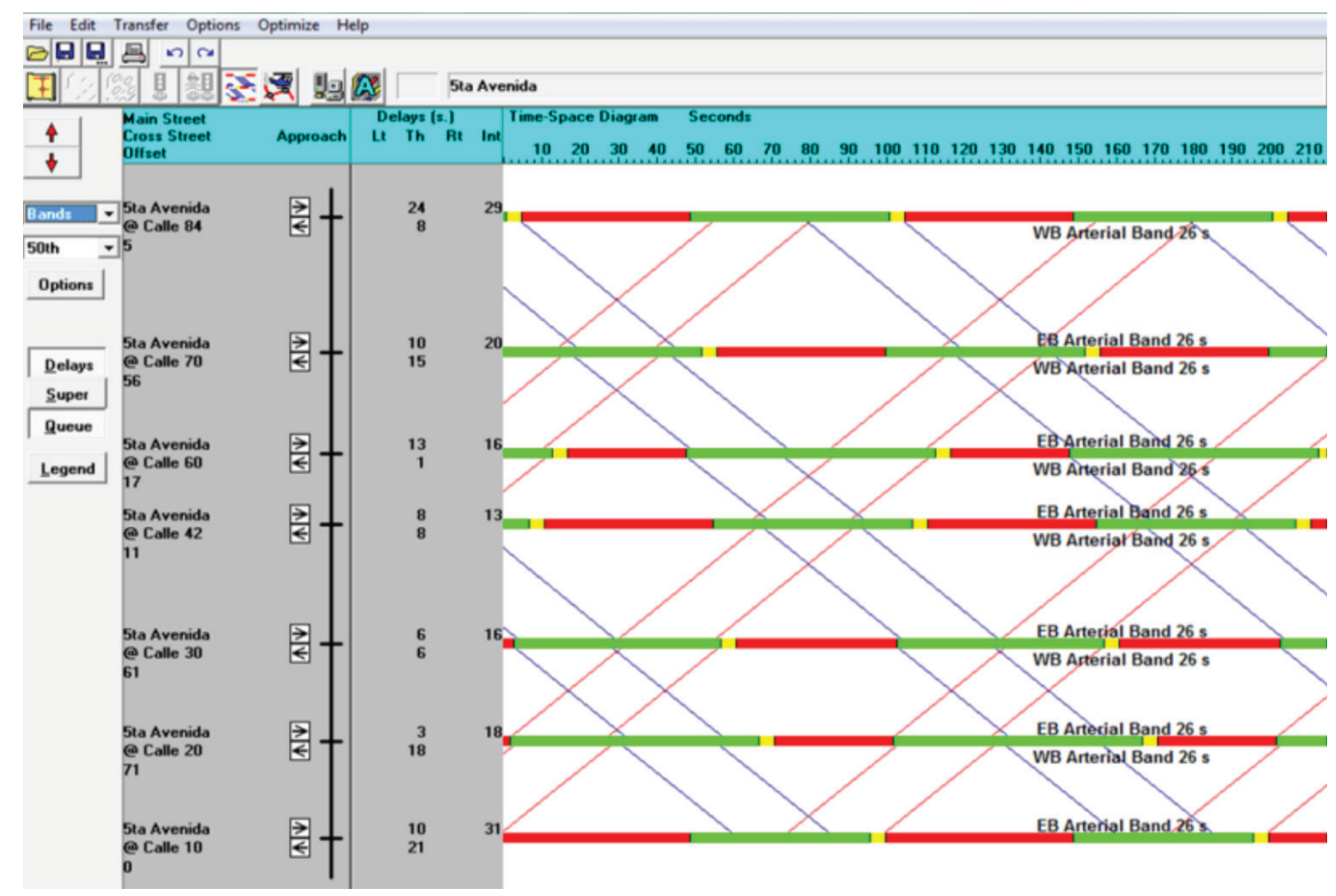

Figura 2. Diagrama de bandas de la variante definitiva (captura del software) 


\begin{tabular}{|c|c|c|c|c|}
\hline \multirow[b]{2}{*}{ Intersecciones } & \multirow[b]{2}{*}{ Tiempo de verde (s) } & \multirow[b]{2}{*}{$\mathrm{C} / \mathrm{g}$} & 5ta Ave desde el este & 5 ta Ave desde el oeste \\
\hline & & & Calidad de la progresión & Calidad de la progresión \\
\hline 5 ta y 10 & 47 & 1,83 & Favorable & Favorable \\
\hline 5 ta y 20 & 65 & 1,54 & Favorable & Favorable \\
\hline 5 ta y 30 & 54 & 1,85 & Altamente Favorable & Favorable \\
\hline 5 ta y 42 & 52 & 1,92 & Favorable & Altamente Favorable \\
\hline 5 ta y 60 & 65 & 1,54 & Favorable & Favorable \\
\hline 5 ta y 70 & 52 & 1,92 & Favorable & Favorable \\
\hline 5 ta y 84 & 52 & 1,92 & Desfavorable & Favorable \\
\hline
\end{tabular}

La calidad de la progresión es muy buena, lo que indica que existe comodidad y seguridad en la circulación y mide la eficiencia del sincronismo realizado.

\section{3. Índice de comportamiento}

La mejor longitud de ciclo puede ser encontrada calculando el índice de comportamiento. Mientras menor sea el índice de comportamiento, la operación será de mayor calidad, por tanto, serán menores las demoras totales y los vehículos que paran.

Para el caso de la cadena sincronizada según los datos que muestra el software, en el sentido 5ta desde este el índice es 6,6 y 5 ta desde el oeste es 1,3, lo que es un indicador de disminución de las demoras y paradas, aumentando la fluidez del tránsito.

\subsection{Vehículos en la zona de dilema}

Este parámetro cuantifica el número de vehículos que arriban mientras el semáforo muestra la luz amarilla, que son los que más probabilidad tienen de tener un accidente de tránsito. Normalmente este parámetro se utiliza para mostrar que la coordinación es una mejora de la seguridad del tránsito (Trafficware, 2017). El Cuadro 8 muestra el número de vehículos en la zona de dilema antes y después de sincronizada la arteria, además de la variación en porciento (relación de vehículos antes y después de la sincronización) de los vehículos en la zona de dilema.

Cuadro 8. Vehículos en la zona de dilema

\begin{tabular}{|c|c|c|c|c|c|c|}
\hline \multirow{2}{*}{ Inter. } & \multicolumn{3}{|c|}{ 5ta Ave desde el este } & \multicolumn{3}{|c|}{ 5ta Ave desile el oeste } \\
\hline & Sin sincronismo & Sincronizada & Variación (\%) & Sin sincronismo & Sincronizada & Variación (\%) \\
\hline 5 ta y 10 & 63 & 50 & 20,63 & 145 & 9 & 93,79 \\
\hline 5 ta y 20 & 80 & 13 & 83,75 & 143 & 21 & 85,31 \\
\hline 5 ta y 30 & 8 & 6 & 25,00 & 19 & 1 & 94,74 \\
\hline 5 ta y 42 & 36 & 2 & 94,44 & 7 & 52 & $-642,86$ \\
\hline 5 ta y 60 & 24 & 6 & 75,00 & 76 & 6 & 92,11 \\
\hline 5 ta y 70 & 144 & 15 & 89,58 & 145 & 11 & 92,41 \\
\hline 5 ta y 84 & 95 & 4 & 95,79 & 83 & 70 & 15,66 \\
\hline
\end{tabular}




\begin{tabular}{|c|c|c|c|c|c|c|c|c|c|}
\hline \multirow[b]{2}{*}{ Intersección } & \multirow[b]{2}{*}{ Accesos } & \multicolumn{3}{|c|}{ Demora (s/veh) } & \multicolumn{2}{|c|}{ NS } & \multicolumn{3}{|c|}{$v / c$} \\
\hline & & $\begin{array}{c}\text { Sin } \\
\text { sincronismo }\end{array}$ & Sincronizada & Variación (\%) & $\begin{array}{c}\text { Sin } \\
\text { sincronismo }\end{array}$ & Sincronizada & $\begin{array}{c}\text { Sin } \\
\text { sincronismo }\end{array}$ & Sincronizada & Variación (\%) \\
\hline \multirow{2}{*}{5 ta y 10} & Desde este & 20,1 & 21,3 & $-5,97$ & $C$ & C & 0,68 & 0,59 & 13,24 \\
\hline & Desde oeste & 25,7 & 9,9 & 61,48 & C & A & 0,86 & 0,72 & 16,28 \\
\hline \multirow{2}{*}{5 ta y 20} & Desde este & 11,3 & 17,7 & $-56,64$ & B & B & 0,82 & 0,41 & 50,00 \\
\hline & Desde oeste & 40 & 2,8 & 93,00 & $\mathrm{D}$ & $A$ & 0,87 & 0,53 & 39,08 \\
\hline \multirow{2}{*}{5 ta y 30} & Desde este & 6,6 & 5,6 & 15,15 & A & A & 0,79 & 0,54 & 31,65 \\
\hline & Desde oeste & 18,4 & 5,6 & 69,57 & B & $\mathrm{A}$ & 0,85 & 0,53 & 37,65 \\
\hline \multirow{2}{*}{5 ta y 42} & Desde este & 22,8 & 8,5 & 62,72 & C & A & 0,74 & 0,54 & 27,03 \\
\hline & Desde oeste & 25,1 & 7,6 & 69,72 & C & A & 0,8 & 0,66 & 17,50 \\
\hline \multirow{2}{*}{5 ta y 60} & Desde este & 31,1 & 5 & 82,44 & C & A & 0,79 & 0,49 & 37,97 \\
\hline & Desde oeste & 24,7 & 13,2 & 46,56 & C & B & 0,92 & 0,55 & 40,22 \\
\hline \multirow{2}{*}{5 ta y 70} & Desde este & 17,4 & 15,1 & 13,22 & B & B & 0,87 & 0,77 & 11,49 \\
\hline & Desde oeste & 27,5 & 9,5 & 65,45 & C & A & 0,84 & 0,67 & 20,24 \\
\hline \multirow{2}{*}{5 ta y 84} & Desde este & 31,5 & 8,5 & 73,02 & C & A & 0,85 & 0,48 & 43,53 \\
\hline & Desde oeste & 95,4 & 24,3 & 74,53 & $\mathrm{~F}$ & $C$ & 1,14 & 0,81 & 28,95 \\
\hline
\end{tabular}

Después del sincronismo, los vehículos en la zona de dilema disminuyen considerablemente (en promedio disminuyeron 57 vehículos en el acceso desde el este y 72 desde el oeste) lo que representa un aumento de la seguridad vial, disminución de las demoras, consumo de combustible (Molina-Navarro et al., 2016) y el riesgo de accidentes sería menor. En el Cuadro 9 se muestran los parámetros de eficiencia: demora, nivel de servicio y relación v/c antes y después de la sincronización de las intersecciones en ambos sentidos.

Analizando los parámetros de eficiencia demora, nivel de servicio y relación v/c en la arteria antes y después de ser sincronizada, se puede concluir que las demoras disminuyen en promedio 11,6 s/veh en el sentido desde el este y 16,2 s/veh para el sentido desde el oeste. Se mejoran los niveles de servicio de cada intersección en los dos sentidos, para el sentido desde el este $57,1 \%$ de las intersecciones pasaron de nivel de servicio C o D para A y en el sentido desde el oeste 71,5\% tienen niveles de servicio A. La relación v/c en el sentido desde el este disminuyó en promedio 0,25 y desde el oeste fue de 0,20.

Los resultados obtenidos muestran cómo la coordinación de la cadena de semáforos en la arteria 5ta Avenida optimiza los parámetros de tráfico, ocasionando una disminución de las demoras y las paradas de vehículos, niveles de servicio de altísima calidad, lo que consecuentemente disminuye el consumo de combustible y las emisiones de contaminantes.

\section{CONCLUSIONES}

- Con la sincronización de la cadena de semáforos en 5ta Avenida se optimizaron los parámetros del tránsito de la arteria y de la mayoría de las intersecciones por separado. Se mejoran los niveles de servicio, disminuyen las demoras, paradas de vehículos, disminuye el número de vehículos en la zona de dilema, emisión de contaminantes, entre otros.

- La coordinación provoca una disminución de más de 350 vehículos en la zona de dilema de toda la arteria.

- Los niveles de servicio de la arteria pasan de D y E para $\mathrm{B}$, se disminuyen más del $50 \%$ de las demoras que existen actualmente y la velocidad arterial aumentó en más de $5 \mathrm{~km} / \mathrm{h}$.

- La sincronización de los semáforos fue más favorable para el sentido desde el oeste, donde en media se disminuye la demora en 16,2 s/veh, más del 70\% de las intersecciones pasan para el nivel de servicio A y la media de la relación $\mathrm{v} / \mathrm{c}$ disminuyó en promedio 0,25 . 


\section{REFERENCIAS}

Alba, M.L. (2008). Procedimiento para el tratamiento de los emplazamientos urbanos con alta concentración de accidentes (Tesis Doctoral). CUJAE, Cuba.

de Araújo, A. y de Castro Neto, M. M. (2018). Calibração do modelo de aceitação de brechas em interseções urbanas com o microssimulador de tráfego VISSIM. Transportes, 26(3), 25-38. doi: 10.14295/transportes.v26i3.1603

Araujo, D.R., Jacondino, G. y Cybis, H.B. (2004). Método de calibração de modelos de micro-simulação de tráfego através de otimização multivariada. Transportes, 12(1), 5-12. doi: 10.14295/transportes.v12i1.122

Advanced Traffic Analysis Center (2003). Signal Coordination Strategies Final Report. Recuperado de: https://www.atacenter.org/ programs/ops/pdf/GFSigCoord.pdf

Benekohal, R.F., Elzohairy, Y.M. y Saak, J.E. (2001). Comparison of delays from HCS, Synchro, PASSER II, PASSER IV and CORSIM for an urban arterial. Recuperado de: http://hdl.handle.net/2142/46347

Bezerra, B.S. (2007). Semáforos: Gestão Técnica, Percepção do Desempenho, Duração dos Tempos (Tesis Doctoral). Universidade de São Paulo, São Carlos.

Cal y Mayor, R. y Cárdenas, J. (2010). Ingeniería de tránsito: Fundamentos y aplicaciones. 8va Edición. México: Editorial Félix Varela.

Cardoso Espinosa, E. y Moreno, V. (2012). Análisis y diseño de software para la sincronización de intersecciones semafóricas. Ingeniería Electrónica, Automática y Comunicaciones, 33(1), 16-24.

CNIT. (2008). Justificación para los semáforos de ciclos ajustados. La Habana: Centro Nacional de Ingenieria del tránsito, MININT.

CNIT. (2010). Sistema Centralizado de control del tránsito en La Habana. La Habana: Centro Nacional de Ingenieria del tránsito, MININT.

Díaz, M., Díaz, J., Ferreiro, J., Pérrez, M., Serran, M., Tomás, R., Sentana, I. y Sentana, E. (2002). Métodos geométricos de coordinación reguladas por semáforos. XIV Congreso Internacional de Ingeniería Gráfica, Santander, España.

Díaz, M.C. y Díaz, J.F. (2002). Métodos geométricos de coordinación de intersecciones reguladas por semáforos. XIV Congreso Internacional de Ingeniería Gráfica, Santander, España.

Espinoza Mondragón, J., Jiménez García, J., Medina Flores, J., y Yáñez Contreras, P. (2015). Sincronización de seis semáforos paralelos mediante simulación con promodel y su efecto en el medio ambiente. Congreso Internacional de Investigación Academia Journals. Academia Journals, Celaya.

Gettman, D., y Head, L. (2003). Surrogate Safety Measures From Traffic Simulation Models Final Report. Recuperado de: https:// www.fhwa.dot.gov/publications/research/safety/03050/

Gil, A. (2012). Valoraciones del comportamiento de la vialidad y el tránsito en la Habana - situación actual. Reunión nacional de jefes de ingeniería de tránsito, Centro Nacional de Ingeniería de Tránsito, Villa Clara.

Hajbabaie, A., Medina, J.C. y Bnekohal, R.F. (2011). Traffic Signal Coordination and Queue Management in Oversaturated Intersection. Recuperado de: https://rosap.ntl.bts.gov/view/dot/20576

Jiménez García, J. Medina Flores, J., y Figueroa Fernández, V. (2015). Simulación con promodel para la sincronización de semáforos. Congreso Internacional de Investigación Academia Journals. Academia Journals, Tabasco.

Koonce, P., Rodegerdts, L., Lee, K., Quayle, S., Beaird, S., Braud, C., Bonneson, J., Tarnoff, P. y Urbanik, T. (2008). Traffic Signal Timing Manual. Washington, DC, FHWA. 
Mahmud, S.M.S., Ferreira, L., Hoque, M.S. y Tavassoli, A. (2019). Micro-simulation modelling for traffic safety: A review and potential application to heterogeneous traffic environment. IATSS Research, 43(1), 27-36. doi: 10.1016/j.iatssr.2018.07.002

Molina-Navarro, A., Zamora-Castro, S., Remess-Pérez, M., y Lagunes-Lagunes, E. (2016). Los semáforos inteligentes en la logística urbana sustentable. Revista de Aplicaciones de la Ingenieria, 3(9), 26-33.

Nyame-Baafi, E., Adams, C.A. y Osei, K.K. (2018). Volume warrants for major and minor roads left-turning traffic lanes at unsignalized t-intersections: a case study using VISSIM modelling. Journal of Traffic and Transportation Engineering (English Edition), 5(5), 417-428. doi: 10.1016/j.jtte.2018.01.005

Osorio, C., Chen, X., Marsico, M., Talas, M., Gao, J. y Zhang, S. (2015). Reducing gridlock probabilities via simulation-based signal control. Transportation Research Procedia, 6, 101-110. doi: 10.1016/j.trpro.2015.03.009

Park, B. y Chen, Y. (2010). Quantifying the benefits of coordinated actuated traffic signal systems: A case study. Recuperado de http:// www.virginiadot.org/vtrc/main/online_reports/pdf/11-cr2.pdf

Pirez, J. (2000). Análisis del comportamiento del volumen del tránsito actual con respecto a 1988. Taller de Ingeniería de Tránsito, La Habana, Cuba.

Shaaban, K. y Radwan, E. (2005). A calibration and validation procedure for microscopic simulation model: a case study of simtraffic for arterial streets. TRB Annual Meeting. Transportation Research Board, National Research Council, Washington.

Tianzi, C., Shaochen, J. y Hongxu, Y. (2013). Comparative study of vissim and sidra on signalized intersection. Procedia - Social and Behavioral Sciences, 96, 2004-2010. doi: 10.1016/j.sbspro.2013.08.226

Traffic Engineering Division. (2005). Traffic signal coordination planning effort. Traffic Engineering, Colorado

Trafficware, LCC (2017). Synchro studio 10: Synchro Plus Simtraffic and 3D viewer (No. Synchro Studio 10 User Guide. Gillingham, Trafficware, LCC.

Transportation Research Board (2010). Highway Capacity Manual, Washington DC, TRB.

Webster F. V. (1958). Traffic Signal Settings. London: Her Majesty’s Stationery Office. 